\title{
Botulinum A Toxin Intravesical Injections in the Treatment of Painful Bladder Syndrome: A Pilot Study
}

\author{
Antonella Giannantoni ${ }^{a, *}$, Elisabetta Costantini ${ }^{a}$, Savino M. Di Stasi ${ }^{c}$, \\ Maria Cristina Tascini ${ }^{a}$, Vittorio Bini $^{b}$, Massimo Porena $^{a}$ \\ a Department of Urology, University of Perugia, Italy \\ ${ }^{\mathrm{b}}$ Department of Internal Medicine, Paediatric Section, University of Perugia, Italy \\ 'Department of Urology, "Tor Vergata" University of Rome, Italy
}

\section{Article info}

Article history:

Accepted December 5, 2005

Published online ahead of

print on January 4, 2006

Keywords:

Botulinum toxin A

Bladder

Painful bladder syndrome

\begin{abstract}
Objective: We evaluated the efficacy and tolerability of botulinum A toxin (BTX-A) intravesical injections in patients affected by painful bladder syndrome with increased urinary frequency, refractory to conventional treatment modalities.

Methods: Twelve women and two men were prospectively included in the study. Under short general anaesthesia patients were given injections of $200 \mathrm{U}$ of commercially available BTX-A diluted in $20 \mathrm{ml} 0.9 \%$ $\mathrm{NaCl}$. Injections were performed submucosally in the trigone and bladder floor under cystoscopic control. Voiding chart, the Visual Analog Scale (VAS) for pain, and urodynamics were performed before treatment and 1 and 3 mo afterward.

Results: Overall, 12 patients (85.7\%) reported subjective improvement at 1 and 3 mo follow-up. The mean VAS score was significantly reduced at 1 and 3 mo after treatment ( $p<0.05$ for both); at the same time points daytime and nighttime urinary frequency significantly decreased $(p<0.01$ and $p<0.05$, respectively), and bladder cystometric capacity significantly increased $(p<0.01)$. Two patients reported incomplete bladder emptying. We did not detect any systemic side effects during or after treatment.

Conclusions: The results of this pilot study indicate that BTX-A intravesical injections are effective in the short-term management of painful bladder syndrome. By modulating afferent C-fiber activity within the bladder walls, BTX-A significantly improves urodynamic parameters and reduces bladder pain and urinary frequency.

(C) 2005 Elsevier B.V. All rights reserved.

\footnotetext{
* Corresponding author. Department of Urology, University of Perugia, Policlinico Monteluce, Via Brunamonti 51, 06100 Perugia, Italy. Tel. +39 075 5793979; Fax: +39 0755726123. E-mail address: agianton@tin.it (A. Giannantoni).
} 


\section{Introduction}

Painful bladder syndrome is characterized by suprapubic pain related to bladder filling, coupled with additional symptoms, such as increased daytime and nighttime urinary frequency, without proven urinary infection or other obvious pathology [1]. The disease is chronic and highly debilitating and has a great negative impact on the quality of life of patients. Several causes have been postulated in the pathogenesis of the condition, including infection, autoimmune response, allergic reaction, neurogenic inflammation, epithelial dysfunction, and inherited susceptibility, but the etiology remains unknown [2]. A number of pharmacologic treatments have been used to treat the pathology; currently, among new potentially effective treatment modalities, botulinum A toxin (BTX-A) intravesical treatment is under investigation.

BTX-A intravesical injections have been demonstrated to be an effective treatment for both neurogenic and nonneurogenic detrusor overactivity $[3,4]$, without producing any consistent axonal sprouting within the detrusor muscle [5].

Recent experimental and clinical evidence suggests also an effect of BTX-A on afferent C fibers, which should explain the antinociceptive effect on bladder tissue in rat chemical cystitis models [6] and in patients with interstitial cystitis [7].

Recently, the ability of a catalytically active derivative of BTX-A to block the secretion from vesicle populations in dorsal root ganglia neuronal cultures has been demonstrated for the release of substance $\mathrm{P}$ and glutamate, which are involved in afferent nociceptive transmission to the central nervous system [8]. This evidence supports the clinical application of BTX-A in the treatment of bladder hypersensitive disorders in humans.

The aim of the present study was to evaluate the efficacy and tolerability of BTX-A intravesical treatment in patients with painful bladder symptoms associated with increased urinary frequency that was refractory to conventional treatment modalities.

\section{Materials and methods}

\subsection{Patients}

The patients enrolled in this prospective study were affected by refractory bladder pain and urgency frequency syndrome, in the presence of sterile urine. The study was approved by the local ethics committee and all patients gave their written informed consent. Patients with myasthenia gravis and other diseases affecting cholinergic action, pregnancy, and concomitant use of aminoglycosides were excluded from the study. Furthermore, we excluded patients with concomitant bladder outlet obstruction, prior radiotherapy, and intravesical stones. Previous treatment modalities, including oral and intravesical therapies, have failed in all patients. Particularly, failing treatments consisted of anticholinergics per os, antidepressants, bladder overdistention, pentosan polysulfate per os, and resiniferatoxin intravesical passive diffusion. In addition, one patient was treated previously with sacral nerve modulation (Table 1).

All patients presented with increased daytime and nighttime urinary frequency (Table 2) and pain in the bladder and urethra, vagina, or perineum during bladder filling or after micturition. Two men, who previously had transurethral resection of bladder tumors and then were treated with intravesical bacillus Calmette-Guérin, complained of severe bladder and perineal pain with urgency-frequency syndrome.

Baseline evaluation included history, physical examination, serum chemistries, urinalyses, urine culture, and imaging assessment of the upper and lower urinary tract. Patients underwent voiding chart, cystoscopy, and urodynamics and used the Visual Analog Scale (VAS) for pain quantification. Urodynamics followed the International Continence Society Standards [9], and it was performed 1 mo before commencing the study.

\subsection{Study plan and treatment}

Fourteen patients fulfilling all criteria were selected and included in the study. Under short general anesthesia patients were given injections of $200 \mathrm{U}$ of commercially available BTXA (Allergan, Irvine, CA) diluted in $20 \mathrm{ml} 0.9 \% \mathrm{NaCl}$. Injections were performed through a cystoscope using a flexible needle, submucosally in the trigone and bladder floor. A total of $10 \mathrm{U}$ BTX-A was used per injection site. After BTX-A injections, a 16 Ch Foley indwelling catheter was routinely inserted for $24 \mathrm{~h}$. Patients were discharged after overnight observation and were followed up for bladder voiding and pain. Uroflowmetry with postvoid residual volume measurements was performed after the catheter was removed. Clinical evaluation, VAS score, cystoscopy, and urodynamics were repeated after 1 and 3 mo. Local or systemic side effects were noted during and after treatment.

\subsection{Data analysis}

Statistical analysis was performed using the Friedman, Wilcoxon, and Mann-Whitney tests for nonparametric data. The Bonferroni correction was applied to post hoc multiple comparisons. Statistical significance was set at $p<0.05$. All data analyses were performed using SPSS release 10.1.1 for Windows (SPSS Inc., Chicago, IL, 1999).

\section{Results}

The 12 women and 2 men had a mean age of $64 \pm 9.6$ $\mathrm{yr}$ and mean disease duration of $3.7 \pm 2.2 \mathrm{yr}$.

Before treatment, mean daytime and nighttime urinary frequencies were $14.2 \pm 3.8$ and $4.5 \pm 1.3$, 
Table 1 - Previous treatments in 14 patients affected by bladder hypersensitive disorders and treated with BTX-A intravesical injections

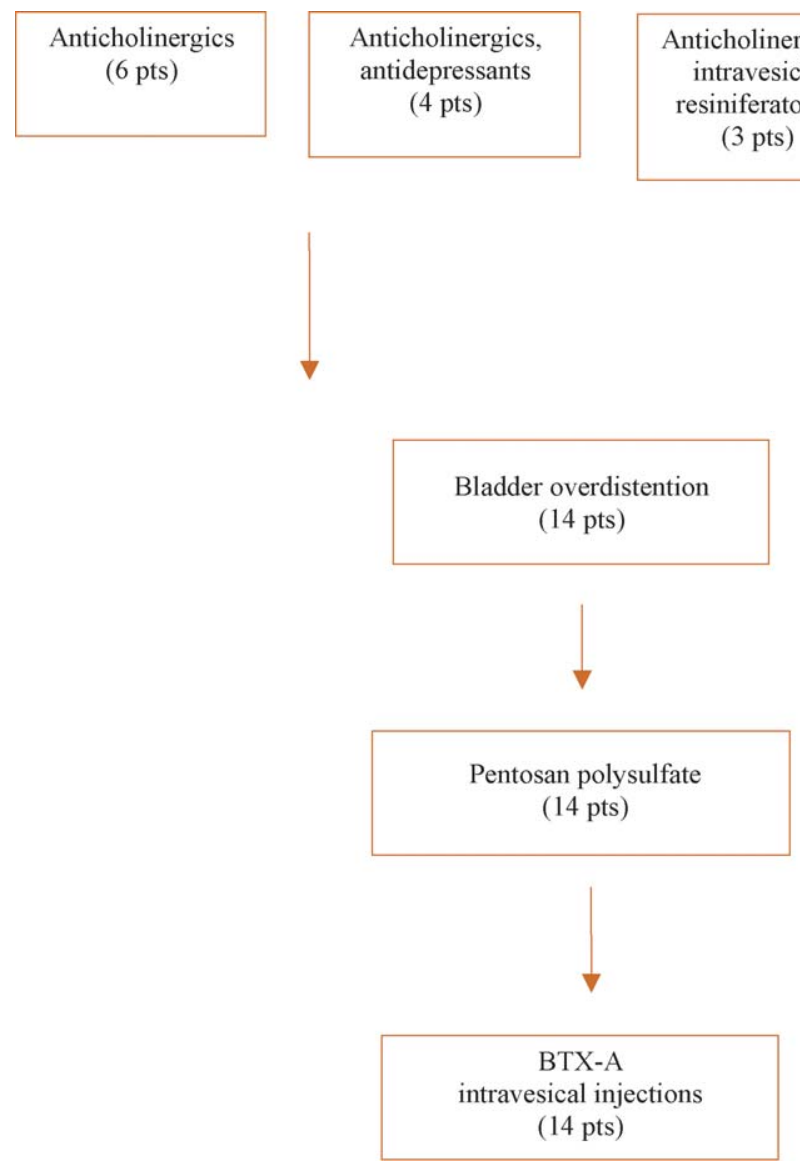

respectively, and the mean VAS score was $9.3 \pm 0.9$. On urodynamics, all patients demonstrated abnormal sensation (first desire $<150 \mathrm{ml}$ ) in the absence of detrusor overactivity. Mean cystometric capacity was $261.6 \pm 34.8 \mathrm{ml}$; no patient had impaired detrusor contractility. Twenty-four hours after BTX-A treatment, 2 patients ( 1 man and 1 woman) needed complementary intermittent catheterization, due to large postvoid residual volume.

One month after therapy, mean daytime and nighttime urinary frequencies were significantly reduced $(8.4 \pm 1.9$ and $2.1 \pm 1.5 ; \quad p<0.01$ and $p<0.05$, respectively). Twelve patients (85.7\%) reported a subjective improvement in painful bladder symptoms; globally, the mean VAS score was $5.8 \pm 2.4(p<0.01)$. Ten patients complained of different grades of dysuria and needed abdominal straining to completely void their bladder. Furthermore, statistically significant improvements in urodynamic parameters were registered. The mean value of maximum cystometric capacity increased from $261.6 \pm 34.8$ to $359.7 \pm 48.4 \mathrm{ml}(p<0.01)$. Globally, we could detect a significant reduction in detrusor pressure at maximum flow rate and in

Table 2 - Clinical and urodynamic results before and 1 and 3 mo after intravesical injections of BTX-A in patients affected by painful bladder syndrome

\begin{tabular}{|c|c|c|c|c|}
\hline Clinical and urodynamic parameters & Baseline (mean $\pm \mathrm{SD}$ ) & 1 mo $($ mean $\pm \mathrm{SD})$ & 3 mo $($ mean $\pm S D)$ & $p$ level \\
\hline Daytime urinary frequency & $14.2 \pm 3.8$ & $8.4 \pm 1.9$ & $9.1 \pm 2.3$ & 0.01 \\
\hline Nighttime urinary frequency & $4.5 \pm 1.3$ & $2.1 \pm 1.5$ & $2.4 \pm 1.7$ & 0.05 \\
\hline VAS score & $9.3 \pm 0.9$ & $5.8 \pm 2.4$ & $6.1 \pm 2.8$ & 0.01 \\
\hline Cystometric bladder capacity & $261.6 \pm 34.8$ & $359.7 \pm 48.4$ & $341.7 \pm 52.4$ & 0.01 \\
\hline pDetQmax & $23 \pm 17.8$ & $15.7 \pm 12.5$ & $17.4 \pm 10.7$ & 0.05 \\
\hline Qmax & $24.2 \pm 4.6$ & $14.6 \pm 13.1$ & $16.9 \pm 9.8$ & 0.01 \\
\hline
\end{tabular}


maximum flow rate at 1 and 3 mo follow-up; in 2 men and 4 women, we observed unsustained detrusor contractions with severe abdominal straining and intermittent urinary flow during the emptying phase, indicating an impaired detrusor contractility.

Clinical and urodynamic results are shown in Table 2. Three months after treatment, the mean VAS score was $6.1 \pm 2.8(p<0.01) ; 12$ patients reported again a consistent improvement in their symptoms, but in 4 patients dysuria persisted. Two patients persisted with complementary intermittent self-catheterization. Mean daytime and nighttime urinary frequencies were $9.1 \pm 2.3$ and $2.4 \pm 1.7$, respectively $(p<0.01$ and $p<0.05)$. Maximum cystometric capacity was substantially similar to that detected at 1 mo follow-up. We did not observe any systemic side effects during or after treatment. All patients were interviewed by telephone at $5 \mathrm{mo}$ follow-up. Bladder pain had recurred in 10 patients and the mean VAS score was $8.6 \pm 0.2$. Two patients complained of dysuria and one was still using intermittent self-catheterization.

\section{Discussion}

Apart from its therapeutic effects to block both striated and smooth muscle contractions, increasing evidence has shown that BTX-A also has analgesic properties in animals and humans. A peripheral antinociceptive effect of the neurotoxin has been suspected on the basis of the reduction in pain associated with migraine headaches, spasmodic torticollis, and chronic myofascial pain [10-12]. Welch et al. observed that various botulinum toxins inhibited release of neuropeptides from rat dorsal root ganglia [13], and Jabbari et al. reported that multiple subcutaneous BTX-A injections relieved segmental burning pain [14].

Few studies revealed the benefits of BTX-A injections in patients suffering from the urgencyfrequency syndrome or interstitial cystitis. Zermann et al. showed reduced frequency and increased bladder capacity in 4 of 7 patients with refractory urgency-frequency syndrome who received intravesical Botox targeting the trigone and bladder base [15]. A recent multicenter case series examined the effect of intravesical Botox or Dysport in 13 patients with refractory interstitial cystitis [16]. Overall, 9 of 13 patients $(69 \%)$ reported a subjective improvement that lasted a mean of 3.72 mo (range, 1-8 mo).

The present study shows that intravesical BTX-A injection successfully relieved pain for a few months in most patients with painful bladder syndrome. Exactly how BTX-A works in the bladder is as yet unclear. Cholinergic blockade at the neuromuscular junction does not account for such sensory effects. Maggi et al. showed that bladder sensory nerves have dual afferent and efferent nerve functions [17]. The sensory efferent actions include the release of transmitters such as adenosine triphosphate (ATP), substance $\mathrm{P}$, and calcitonin gene-related peptide (CGRP) that can act on nearby tissues and on afferent nerve terminals in an autocrine fashion to increase afferent nerve activity [17]. The production and release of these neurotransmitters increase during conditions of inflammation and pain $[7,16]$. Moreover, many $C$ fibers in the bladder mucosa contain neuropeptides, such as substance $\mathrm{P}$, neurokinin $\mathrm{A}$, and CGRP, which on release, can modulate the micturition reflex and might cause detrusor overactivity [18].

Another neurotransmitter involved in inflammation is ATP. Recently, it has been observed that BTXA inhibits ATP release from bladder urothelium in rats [18], and increased stretch activated ATP release has been reported from human urothelial cells cultured from the bladders of patients with interstitial cystitis and spinal cord injury [19].

Finally, it has been observed that BTX-A intravesical injections reduce nerve growth factor (NGF) bladder tissue content in patients with spinal cord injury (SCI) patients with detrusor overactivity [20]. NGF, which is increased not only after SCI, but also in inflamed tissues and in serum of patients with inflammatory conditions, has been shown to act on trkA receptors to mediate substance $\mathrm{P}$ biosynthesis and release from afferent neurons [21]. Once released from afferent terminals, substance $P$ acts on NK1 receptors and mediates neurogenic inflammation consisting of increased vascular permeability, plasma extravasation, glandular secretion, and proinflammatory cell influx.

Indeed, the mechanisms underlying the antinociceptive effect of BTX-A may be more complex because recent observations revealed that human urothelium also receives cholinergic innervation and can release acetylcholine at rest [22]. This nonneural acetylcholine could bind to muscarinic receptor populations within urothelium or afferent nerves, thereby modulating bladder sensory input to the central nervous system.

In the present study, the significant increase in bladder capacity and the reduction in urinary frequency, as observed at 1 and 3 mo follow-up, can be explained by the ability of BTX-A to spread into close tissues. Thus, at least the lateral walls of the bladder could have been influenced by the action of the neurotoxin. Indeed, the decrease in sensory input from the bladder to the central nervous system could 
explain the reduction in frequency. Moreover, the relief of pain itself may have been an adjunctive factor supporting frequency amelioration.

One major concern in the present study was that BTX-A impaired detrusor contractility in 6 of 14 patients, even though it was administered submucosally. Indeed, incomplete bladder emptying represents a serious complication in nonneurogenic patients who are not used to performing selfcatheterization. BTX-A may have spread into underlying tissues, thus reaching muscarinic receptors in the detrusor muscle. In future studies this complication should be avoided by reducing the dosage or dilution or by using intravesical instillation, which can be administered without general anesthesia. However, because the molecular weight of BTX-A is $150 \mathrm{kD}$, penetrating the intact urothelium is difficult and mucosal permeability may need to be increased before administering BTX-A. This approach would prevent BTX-A from reaching the muscle layer and limit its action to the bladder mucosa, thus relieving bladder pain without modifying detrusor contractility.

One final point is worth mentioning. Because the effects of BTX-A toxin are transient, repeated doses, as indeed our patients requested, are needed for effective control of bladder pain and urgency. The dose with the longest duration and lowest risk of urinary retention still needs to be established.

\section{Conclusions}

The results of this pilot study indicate that BTX-A intravesical injections are effective in the shortterm management of painful bladder syndrome. By modulating afferent C-fiber activity within the bladder walls, BTX-A significantly improves urodynamic parameters and reduces bladder pain and urinary frequency. The injection of the neurotoxin into the bladder wall impairs detrusor contractility in a minority of patients. No systemic side effects were observed during and after treatment. Further research is needed to explore the underlying mechanisms (eg, neuronal, afferent, or efferent) of analgesia induced by BTX-A.

\section{References}

[1] Chancellor MB, Yoshimura N. Treatment of interstitial cystitis. Urology 2004;63(suppl 3A):85-92.

[2] Diokno AC, Homma Y, Sekiguchi Y, Suzuki Y. Interstitial cystitis, gynaecologic pelvic pain, prostatitis, and their epidemiology. Int J Urol 2003;10:S3-6.
[3] Popat R, Apostolidis A, Kalsi V, Gonzales G, Fowler CJ, Dasgupta P. A comparison between the response of patients with idiopathic detrusor overactivity and neurogenic detrusor overactivity to the first intradetrusor injection of botulinum A toxin. J Urol 2005;174:984-9.

[4] Reitz A, Stohrer M, Kramer G, Del Popolo G, Chartier-Kastler E, Pannek J, et al. European experience of 200 cases treated with botulinum-A toxin injections into the detrusor muscle for urinary incontinence due to neurogenic detrusor overactivity. Eur Urol 2004;45:510-5.

[5] Haferkamp A, Schurch B, Reitz A, Krengel U, Grosse J, Kramer G, et al. Lack of ultrastructural detrusor changes following endoscopic injection of botulinum toxin type A in overactive neurogenic bladder. Eur Urol 2004;46:784-91.

[6] Cayan S, Coskun B, Bozlu M, Acar D, Akbay E, Ulusoy E. Botulinum toxin type A may improve bladder function in a rat chemical cystitis model. Urol Res 2003;30:399-404.

[7] Smith CP, Chancellor MB. Emerging role of botulinum toxin in the management of voiding dysfunction. J Urol 2004;171:2128-37.

[8] Duggan MJ, Quinn CP, Chaddock JA, Purkiss JR, Alexander FCG. Doward S, et al. Inhibition of release of neurotransmitters from rat dorsal root ganglia by a novel conjugate of a clostridium botulinum toxin A endopeptidase fragment and erythrina cristagalli lectin. J Biol Chem 2002;277:34846-52.

[9] Abrams P, Cardozo L, Fall M, Griffiths D, Rosier P, Ulmsten $\mathrm{U}$, et al. The standardisation of terminology of lower urinary tract function: report from the standardisation subcommittee of the International Continence Society. Neurourol Urodyn 2002;21:167.

[10] Tsui JK, Eisen A, Stoessl AJ, et al. Double-blind study of botulinum toxin in spasmodic torticollis. Lancet 1986;2: 245-7.

[11] Cheshire WP, Abashian SW, Mann JD. Botulinum toxin in the treatment of myofascial pain syndrome. Pain 1994;59: 65-9.

[12] Silberstein B, Maher N, Difasio MP. Botulinum toxin type A as a migraine preventive treatment. Headache 2000;40: 445-50.

[13] Welch MJ, Purkiss JR, Foster KA. Sensitivity of embryonic rat dorsal root ganglia neurons to Clostridium botulinum neurotoxins. Toxicon 2000;38:245-8.

[14] Jabbari B, Maher N, Difazio MP. Botulinum toxin-A improved burning pain and allodynia in two patients with spinal cord pathology. Pain Med 2003;4:206-8.

[15] Zermann DH, Ishigooka M, Schubert J. Trigonum and bladder base injection of severe urgency-frequency syndrome refractory to conservative medical treatment and electrical stimulation. Neurourol Urodyn 2001;20:412-3.

[16] Smith CP, Radziszewski P, Borkowski A, Somogyi GT, Boone TB, Chancellor MB. Botulinum toxin A has antinociceptive effects in treating interstitial cystitis. Urology 2004;64:871-5.

[17] Maggi CA. The dual sensory and efferent functions of the capasaicin-sensitive primary sensory nerves in the bladder and urethra. In: Maggi, C.A., editor. The Autonomic Nervous System: Nervous Control of the Urogenital System, vol. 3. London, Harwood Academic Publisher, London, p. 383. 
[18] Khera M, Somogyi GT, Kiss S, Boone TB, Smith CP. Botulinum toxin A inhibits ATP release from bladder urothelium after chronic spinal cord injury. Neurochem Int 2004;45:987-93.

[19] Sun Y, Keay S, De Deyne PG, Chai TC. Augmented stretch activated adenosine triphosphate release from bladder uroepithelial cells in patients with interstitial cystitis. J Urol 2001;166:1951-6.

[20] Giannantoni A, Di Stasi SM, Nardicchi V, Macchioni L, Guercini F, Goracci G, et al. Botulinum A toxin intravesical treatment induces a reduction of nerve growth factor bladder tissue levels in patients with neurogenic detrusor overactivity. J Urol 2005;173:330.

[21] Dinh QT, Groneberg DA, Peisert C, Springer J, Joachim RA, Arck PC, et al. Nerve growth factor-induced substance $P$ in capsaicin insensitive vagal neurons innervating the lower mouse airway. Clin Exp Allergy 2004;34:1474-9.

[22] Andersson KE, Yoshida M. Antimuscarinics and the overactive detrusor-which is the main mechanism of action. Eur Urol 2003;43:1-5.

\section{Editorial Comment \\ F. Burkhard, University Hospital Bern, Switzerland E-mail address: fiona.burkhard@insel.ch}

Chronic pelvic pain syndrome (CPPS) is an entity of unknown origin that affects a large number of both men ad women. The impact of CPPS on the patients quality of life is immense, with a negative impact on social activities, personal relationships, sexuality and physical activityas well as psychological well being [1].

Following clinical suspicion of CPPS the diagnosis is not as in most diseases confirmed by a positive findings on objective examinations, but rather by an extensive diagnostic work up excluding other urological abnormalities. This because the true pathology behind this symptom complex remains an enigma [2]. Many authors believe in an inflammatory process, however to date a pathogen has not been found. Another potential mechanism is a neuroinflammatory cascade evoking an increased pain response.

The same problem arises when it comes to treating the patient. There are multiple therapeutic options ranging from antibiotic treatment to cystectomy and bladder substitution.

Here a pilot study is presented using botulinum A toxin injections into the trigone and bladder floor for the treatment of CPPS. Eighty-six percent of patients reported a subjective improvement at 1 and 3 months. This further increases the evidence, that Botulinum A toxin has an antinociceptive effect [3]. If this is through an effect on afferent pathways, efferent pathways or urothelial cell activity remains to be determined. Another interesting point is the role of the trigone in CPPS, which remains unclear and published results are controvers $[3,4]$.

Overall the use of botulinum A toxin for treating CPPS is a promising minimally invasive concept. In future better understanding of the pathogenetic mechanism behind CPPS may help us choose the most appropriate treatment option for each individual patient.

\section{References}

[1] Ku JH, Kim SW, Paick JS. Quality of life and psychological factors in chronic prostatitis/chronic pelvic pain syndrome. Urology 2005;66:693.

[2] Moldwin RM, Sant GR. Interstitial cystitis: a pathophysiology and treatment update. Clin Obstet Gynecol 2002;45: 259.

[3] Smith CP, Radziszewski P, Borkowski A, Somogyi GT, Boone TB, Chancellor MB. Botulinum toxin a has antinociceptive effects in treating interstitial cystitis. Urology 2004;64:871.

[4] Kuo HC. Preliminary results of suburothelial injection of botulinum A toxin in the treatment of chronic interstitial cystitis. Urol Int 2005;75:170. 